\title{
Absence of the Aortic Valve Associated with Hypoplastic Left- Sided Heart Syndrome
}

\author{
Edmar A tik, Luiz A lberto Benvenuti, Tamara Cortez Martins, Miguel Barbero-Marcial
}

São Paulo, SP - Brazil

\begin{abstract}
In this report we describe the twelveth case in the literature of absence of the aortic valve cusps, associated with hypoplastic left-sided heart syndrome in a neonate. Clinical and hemodynamic conditions in our patient resemble the classical features of this syndrome except for a greater development of the ascending aorta and the left ventricular cavity, due to aortic insufficiency. A patch was unsuccessfully inserted at the aortic annulus to exclude the left ventricle from the circulation. In addition the Norwood operation was performed.
\end{abstract}

In contrast with the absence of the pulmonary valve ${ }^{1}$, the aortic valve agenesis, which always occurs in association with other defects, is much rarer. Just eleven such cases have been reported in the medical literature ${ }^{2-10}$. In eight of these, associated hypoplastic left-sided heart syndrome was present ${ }^{4-8,10}$. Normally related great arteries with aortic annulus stenosis was present in one ${ }^{9}$ and in another two patients double outlet right ventricle was present ${ }^{2,3}$ (being in one part of a complex anatomy with polisplenia) ${ }^{3}$. Other defects have been described, for example total anomalous venous drainage ${ }^{8}$, pulmonary venous stenosis ${ }^{8}$, subaortic stenosis by anomalous mitral chordal attachments ${ }^{4}$ and left ventricular endocardial fibroelastosis ${ }^{4,5,8,10}$.

We relate one more such case of absence of the aortic valve associated with hypoplastic left-sided heart syndrome, but with a well developed ascending aorta and left ven tricular cavity, due to a prominent aortic insufficiency, both being diagnosed in life and confirmed at autopsy.

\section{Case Report}

Our patient was a male neonate born weighing $4190 \mathrm{~g}$ after an uneventful pregnancy and a normal delivery. He was discharged on the second day, but a mild cyanosis and tachypnea were responsible for his readmission to our service after 22 days of life.

At that time, the baby had weak pulses that improved

Instituto do Coração do Hospital das Clínicas - FMUSP

Mailing address: Edmar Atik - InCor - Av. Dr. Enéas C. Aguiar, 44 - 05403-000 - São Paulo, SP - Brazil after prostaglandin $\mathrm{E}_{1} \mathrm{IV}-0.01 \mu \mathrm{g} / \mathrm{kg} / \mathrm{mm}$. Suddenly, he became acyanotic, with strong pulses, mostly at the lower limbs, with a very comfortable clinical state.

His weight was $4270 \mathrm{~g}$, heart rate was 150 per minute and blood pressure was $111 \times 69 \mathrm{mmHg}$ in the upper extremities and $88 \times 43 \mathrm{mmHg}$ in the lower extremities. Pulse oxygen saturation was 98 at rest and 85 with crying.

Remarkable cardiac findings were impulsive left sternal border lift, accentuated cardiac heart sounds, systolic ejection click all over the precordium and a systolic and diastolic (to and fro) moderate intensity murmur along the left sternal border.

The lungs were clear and the liver was palpable at three centimeters below the right costal margin.

Eletrocardiography revealed sinus rhythm, the QRS axis at $+150^{\circ}$, the $\mathrm{T}$ axis at $+90^{\circ}$ and the $\mathrm{P}$ axis at $+60^{\circ}$. Undetermined ventricular overload was evident as the rS complexes were visible from $\mathrm{V}_{1}$ through $\mathrm{V}_{6}$.

Chest radiography showed moderate cardiac enlargement and increased pulmonary vascular markings.

Laboratory blood analysis revealed: $\mathrm{Hg}: 14.7 \mathrm{~g} \%$, Hct: 44\%, CKMB: 18U, Urea: 29, Creatinine: 0.6mg.

Echocardiography and cardiac catheterization (fig.1) characterized the anomaly in the setting of an absence of left atrioventricular connection with a concordant ventriculoarterial connection. A large reversal ductal flow from huge pulmonary arteries was seen with a retrograde filling of the ascending aorta and subsequently the left ventricular cavity through a remarkable aortic valve insufficiency. A small left atrium received all pulmonary veins that drained to the right atrium through two atrial septal defects of five and $2 \mathrm{~m}$ $m$ in diameter. The left ventricle was small but well formed and the ascending aorta was $8 \mathrm{~mm}$ in diameter. A rudimentary aortic valve was evident at the aortic annulus.

The pressures at cardiac catheterization were: RA: 8, LA: 20, RV: 70/8,LV: 70/8, Ao: 70/30-43mmHg.

The Norwood operation, a termino-lateral pulmonary trunk anastomosis to the ascending aorta with an arterial duct ligation was performed at 29 days of life with extracorporeal circulatory arrest at $20^{\circ} \mathrm{C}$. A striking anatomical feature was the absence of any aortic valve tissue beneath the aortic sinuses, seen by lateral aortotomy, except for a very rudimentary and nonobstructive flat ridge of $1 \mathrm{~mm}$ extension in the posterior aortic annulus. The aorta was about $8 \mathrm{~mm}$ in 


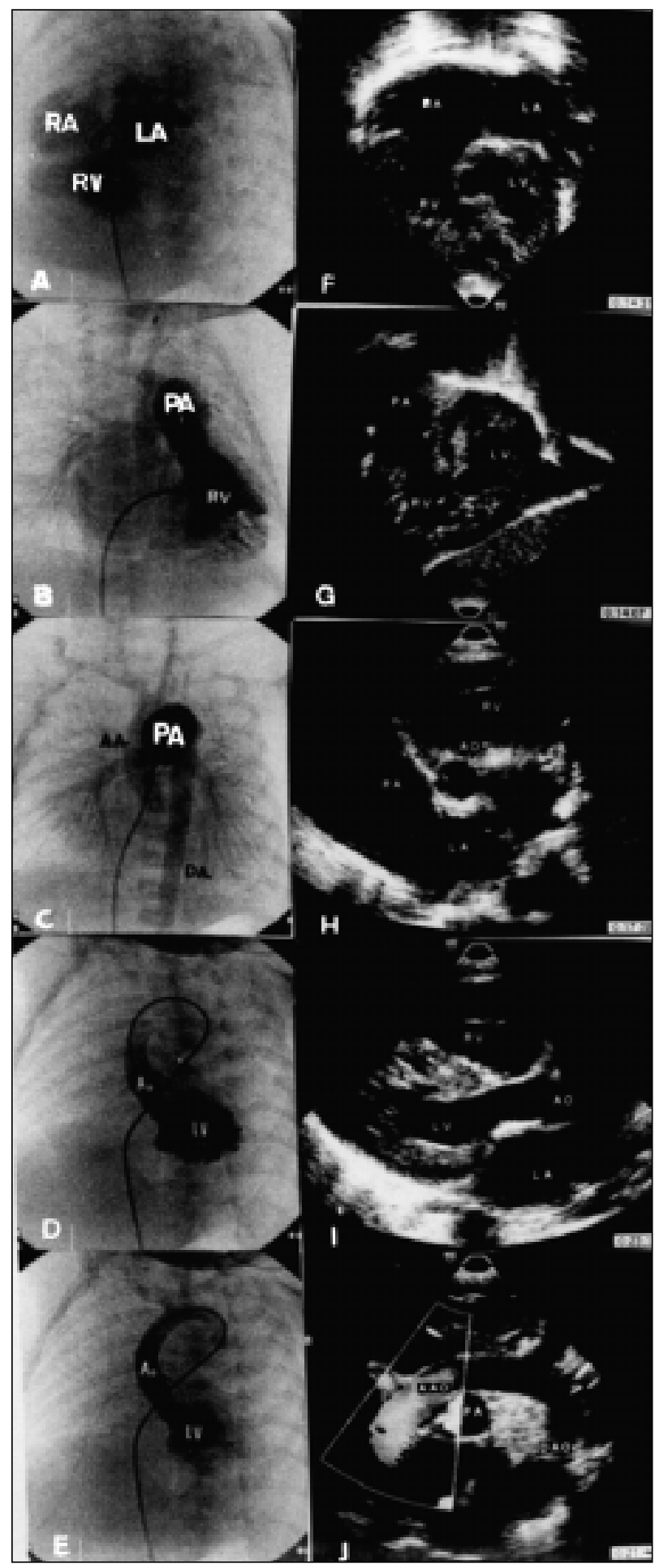

Fig. 1 - Angiographic features correlated well with echocardiographic ones in the setting of association of defects: absence of aortic valve and hypoplastic left heart syndrome. Absence of the left atrioventricular connection with small left atrium (LA) are well seen in (a) and (f). The dilated right ventricular (RV) cavity (b,g) receives all venous return, the systemic and the pulmonary by an atrial septal defect (a) with a concordant ventriculoarterial connection (b,d,g,i). Huge pulmonary arteries (PA) are responsible for maintaining the systemic circulation including ascending aorta (AAo) and coronary arteries by a retrograde route through a large ductus $(b, c)$. Aortic insufficiency was clearly demonstrated $(e, j)$ with a left ventricle (LV) filling from an ascending aorta injection. The left ventricle and ascending aorta were small but well formed and the left ventricle showed a trabeculated aspect (d). Rudimentary and vestigious aortic leaflets are seen at the right posterior wall of the aortic annulus (h). Ao- ascending aorta; Daodescending aorta; RA- right atrium.
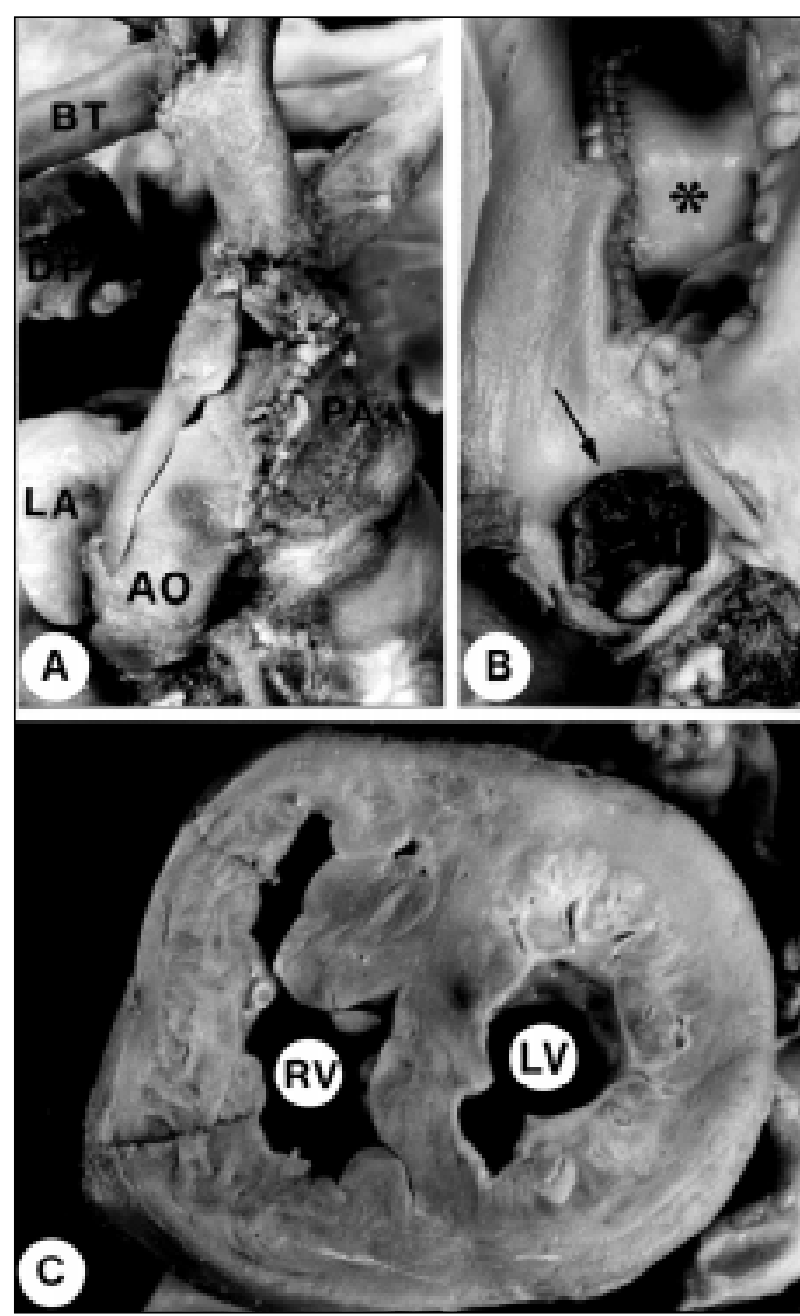

Fig. 2 - Macroscopic view of the heart. A, Right lateral view of the great vessels. The large ascending aorta is apparent (Ao), which was anastomosed to the pulmonary artery (PA). Notice the synthetic Blalock-Taussig shunt (BT), the surgically closed distal pulmonary artery (DPA), and the retroaortic projection of the left atrial appendage (LA). B, Internal view of the ascending aorta. The aortic orifice was closed by a surgical patch (arrow), and a large surgical comunication between the aorta and the pulmonary artery (asterisc) was created. C, The left ventricle has mild hypoplasia and endocardial fibroelastosis (LV). The right ventricle is enlarged and hypertrophied (RV).

diameter, the pulmonary trunk was three times bigger and the patent duct was as large as the pulmonary arteries. On operation, the left ventricular cavity was disconnected from the systemic circulation by a pericardial patch at the site where it should have encountered the aortic leaflets. A modified Blalock-Taussig shunt with a $4 \mathrm{~mm}$ Gore-Tex shunt at the right-sided heart and an atrial septal defect enlargement completed the operation.

Low cardiac output syndrome and hypoxia (pO2: 35 and oxygen saturation: $75 \%$ ) were responsible for death six hours after the operation was completed.

Necropsy showed at the left side of the heart an atretic left atrioventricular valve and a small and hypertrophied left atrium. An anomalous left atrial appendage was present with a bifid shape in a retroaortic projection. The ascending aorta was well developed, measuring $8 \mathrm{~mm}$ in external diameter. The pulmonary trunk was surgically closed and anastomosed to the ascending aorta and to the aortic arch. A syn- 
thetic Blalock-Taussig shunt was interposed between the right subclavian artery and the right pulmonary artery (fig. 2A). The annulus of the aortic valve measured $4 \mathrm{~mm}$ without any vestigious valve, and the aortic orifice was closed with a surgical patch (fig. 2B). The arterial duct was ligated and mild preductal aortic coarctation was present. The valve of the fossa ovalis was surgically removed, and a $12 \mathrm{~mm}$ atrial communication resulted. A mild hypoplasia of the left ventricle was also present, which had endocardial fibroelastosis (fig. 2C). The right cardiac chambers were enlarged and hypertrophied. The coronary arteries originated from the ascending aorta and did not have abnormalities. The histological examination disclosed multiple patchy areas of recent myocardial infarction in both ventricles, chronic pulmonary venous hypertension, with arterial wall hypertrophy and peripheral muscularization and acute tubular necrosis of the kidneys.

\section{Discussion}

Absence of cardiac valves is encountered more in the right-sided heart, mostly at the pulmonary site ${ }^{1}$. The partially unguarded mitral valve is rarely seen, and just eleven cases of aortic valve absence have been mentioned in the literature ${ }^{2-10}$.

In general, the clinical and hemodynamics conditions of unguarded valve annulus are determined by vestigial, severely hypoplastic and nonfunctioning leaflets giving rise to augmented cardiac and vascular structures.

In the absence of the aortic valve, a well developed left ventricle in addition to the ascending aorta become striking features even in the presence of hypoplastic left heart syndrome, mostly demonstrated in the cases described by $\mathrm{Ca}$ brera et al ${ }^{6}$, Harada et $\mathrm{al}^{10}$, Niwa et $\mathrm{al}^{5}$, Lin et al ${ }^{8}$ and by ourselves, where, incidentally, atresia of the left atrioventricular valve was present in all of these cases. The left ventricle was hypoplastic in the seven other cases reported in the li-

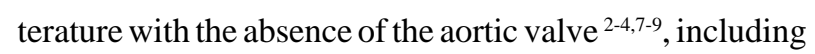
two with a double outlet right ventricle ${ }^{2,3}$. Otherwise, the aortic insufficiency was remarkable for contributing to early death in the first eight days of life in ten cases reported ${ }^{2-9}$.

Although the presence of the unusual left ventricular and ascending aorta development provoked by aortic insufficiency in the case related herein, the clinical picture was similar to that encountered in patients with hypoplastic left-sided heart syndrome in which blood to coronary circulation comes from a retrograde flow from a persistent arterial duct.

The hemodynamic, angiographic and echocardiographic studies were very important in establishing the aortic insufficiency diagnosis, which was suspected clinically by a "to and fro" murmur.

The still challenging Norwood technique was easy to accomplish, in view of a well developed ascending aorta found in this patient. As the left ventricle did not appear to contribute as a systemic pump in this case (it received blood in a retrograde manner) we decided to exclude it from the circulation by putting a patch at the aortic annulus.

Thus, this is just the second absence of aortic leaflet case reported that was treated surgically. Unfortunately, the downhill evolution of our patient was due to a myocardial infarction that occurred during surgery, probably due to the anoxic arrest.

The sole survivor, described by Harada et al ${ }^{10}$, underwent the Norwood technique.

All eleven cases reported occurred in males, which suggests X-linked factors, as has already been previously pointed out ${ }^{8}$.

\section{Acknowledgements}

We thank Dr Vera D. Aiello for pathological assistance, Dr Andressa M. Soares for the echocardiographic analysis, Dr Luis Kajita for the angiographic diagnosis and Dr Munir Ebaid for the clinical discussion.

\section{References}

1. Kirklin JW, Barratt-Boyes BG. Tetralogy of Fallot with absent pulmonary valve. In: Kirklin JW, Barratt-Boyes BG: Cardiac Surgery. $2^{\text {nd }}$ ed. Churchill Livingstone: New York, 1993: 973-5.

2. Towes WH, Lortscher RH, Kelminson LL. Double outlet right ventricle with absent aortic valve. Chest 1975; 68: 381-2.

3. BiermanFZ, Yeh MN, Swersky S, MartinE, Wigger JH, Fox H. Absence of the aortic valve: Antenatal and postnatal two-dimensional and Doppler echocardiographic features. J Am Coll Cardiol 1984; 3: 833-7.

4. RossiMB, HoSY, TaskerRC. Absent aortic valve leaflets. Int JCardiol 1986; 11:235-7.

5. Niwa K, Ikeda F, Miyamoto H, Nakajima H, Ando M. Absent aortic valve with normally related great arteries. Heart Vessels 1987; 3: 104-107.
6. Cabrera A, Galdeano JM, Pastor E. Absence of the aortic valve cusps with mitral atresia, normal left ventricle, and intact ventricular septum. Br Heart J 1990; 63: 187-8.

7. Parikh SR, Hurwitz RA, Caldwell RL, Waller B. Absent aortic valve in hypoplastic left heart syndrome. Am Heart J 1990;119: 977-8.

8. Lin AE, Chin AJ. Absent aortic valve: a complex anomaly. Pediatr Cardiol 1990; 11: $195-8$.

9. Nakamura S, Hatano T. A case of absent aortic valve with severe stenosis. Acta Cardiol Paediatr Jpn 1996; 12: 56-62.

10. Harada Y, Takeuchi T, Satomi G, Yasukouchi S. Absent aortic valve: successful palliation in the neonate. Ann Thorac Surg 1998; 66: 935-6. 